\title{
二维核磁共振谱研究 $3(\mathbf{R}), 8$-二甲基 $-2(R)$, 5-二(1-甲基-乙基)-6-酮-2,3,6,7,9， 10-六氢-1H-3A, 7-乙带-10-腈的立体结构
}

\author{
韩秀文 \\ (中国科学院大连化学物理研究所) \\ H. Rüegger J. Sonderegger \\ (Spectrospin AG, Zürich)
}

关锤词相敏 COSY、相敏 NOESY、远程 C-H化学位移相关徣、BIRD 脉冲

郭永沺等由莪术醇合成草酮类化合物, 进而合成出有明显降压作用的拟生物 碱 新 化 合 物 ${ }^{[1]}$. 其结晶性中间产物的结构确定对鉴定最终还原产物和合成、设计其它改性物十分重要. 寅元素分析和质谱数据确定了此未知物的分子式为 $\mathrm{C}_{21} \mathrm{H}_{28} \mathrm{NO}$; 红外谱表明了酮基、异丙基、 双键和昰基的存在. 本文用同核和异核化学位移相关谱研究了此未知物的立体结构. 成功的 异核化学位移相关谱成为确定立体结构的强有力工具之一，它提供了许多大于一键的 $\mathrm{C}-\mathrm{H}$ 远程偶合信息,特别是具有反式构象的三键 $\mathrm{C}-\mathrm{H}$ 偶合

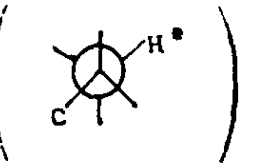
呈现出很强的交叉蜂， 这成为确定相邻官能团、归属同碳氢和季碳的强有力证据; 由高场 'HNMR 谱和模拟谱得到了 全部相邻 ${ }^{1} \mathrm{H}-{ }^{1} \mathrm{H}$ 偶合常数,由其与二面角关系及相敏 NOESY 谱中 NOE 峰, 研究了环和 取代基的构象，其结构确定为 3(R)，8-二甲基-2(R)，5-二(1-甲基-乙基)-6-酮-2，3，6,7， 9,10 -六氢化-1H-3A，7-乙苒-10 腈, 经计算机检索证明为一新化合物. 同时获得了这一新 化合物的金部 ${ }^{t} \mathrm{H},{ }^{13} \mathrm{C}$ NMR 谱线归属和 ${ }^{1} \mathrm{H}-{ }^{1} \mathrm{H}$ 偶合常数.

\section{一、实 验}

化合物的 NMR 谱分别在 Bruker AM-600 ( $\left.{ }^{2} \mathrm{H}: 600.13 \mathrm{MHz}\right)$ 和 AM-400 ('H: 400.13 $\mathrm{MHz}$ ) NMR 谱仪上,在 $27^{\circ} \mathrm{C}$ 下测得。相敏 COSY 脉冲采用 TPPI 和双量子海波 ${ }^{[2]}$, 时域 数据矩阵为 $1 k\left(f_{2}\right) \times 256\left(f_{1}\right)$, 傅里叶变换后在 $F_{1}$ 域零填充产生 $1 k \times 1 k$ 矩阵; NOE 信息 由相敏 NOESY 脉冲序列 ${ }^{[3]}$ 得到, 混合时间 $D$, 为 $0.8 \mathrm{~s}$, 约 $20 \mathrm{~ms}$ 随机变化以消除零量子相 干; $\mathrm{C}-\mathrm{H}$ 相关谱由 $\chi \mathrm{HCORRD}$ [4] 序列测得, 取 ' $J_{\mathrm{CH}}=131 \mathrm{~Hz}$ ，极化转移延迟 $D_{3}-3.8 \mathrm{~ms}$; 远程 C-H $\delta-\delta$ 相关谱由 Martin 脉冲序列 ${ }^{[5-7]}$ 得到, 在混合脉冲后的 $\Delta_{2}$ 延滞中间加一个

本文1988年12月17日收到. 1989 年3 月31 日收到售改稿. 
BIRD 脉冲以等待最住级化而压制一键调制，取 $J_{\mathrm{LR}}-10 \mathrm{~Hz}$ ，远程极化延迟 $D_{2}-50 \mathrm{~ms}$ ， $\Delta_{0}-\frac{1}{2} D_{2}$, 数据矩阵为 $2 k\left(f_{2}\right) \times 512\left(f_{1}\right)$, 在 $F_{1}$ 和 $F_{2}$ 域分别应用末位移的 Sine bell 和 Gausian 窗函数及功率谱; 异核相关谱和 COSY、NOESY 谱分别采用位移的 Sine bell 和 未位移的 Sine bell 窗函数; 用 Panic 程序得其 ' $\mathrm{H}$ 模拟谱.

$15 \mathrm{mg}$ 样品溶解在 $5 \mathrm{ml} \mathrm{CDCl}$, 中, 取 TMS 为化学位移参考, $\mathrm{CDCl}$, 为 ${ }^{13} \mathrm{C} \mathrm{NMR}$ 谱化 学位移的第二标准.

\section{二、结果及讨论}

化合物的 ${ }^{1} \mathrm{H} 、{ }^{13} \mathrm{C}$ 和 DEPT NMR 谱可以清楚地确定酮基、氧基、两个异丙基、一个与 $\mathrm{CH}$-相连的甲基和一个与双键相连的甲基; 解析 COSY 谱(图 1)中相邻质子的相关峰, 可以

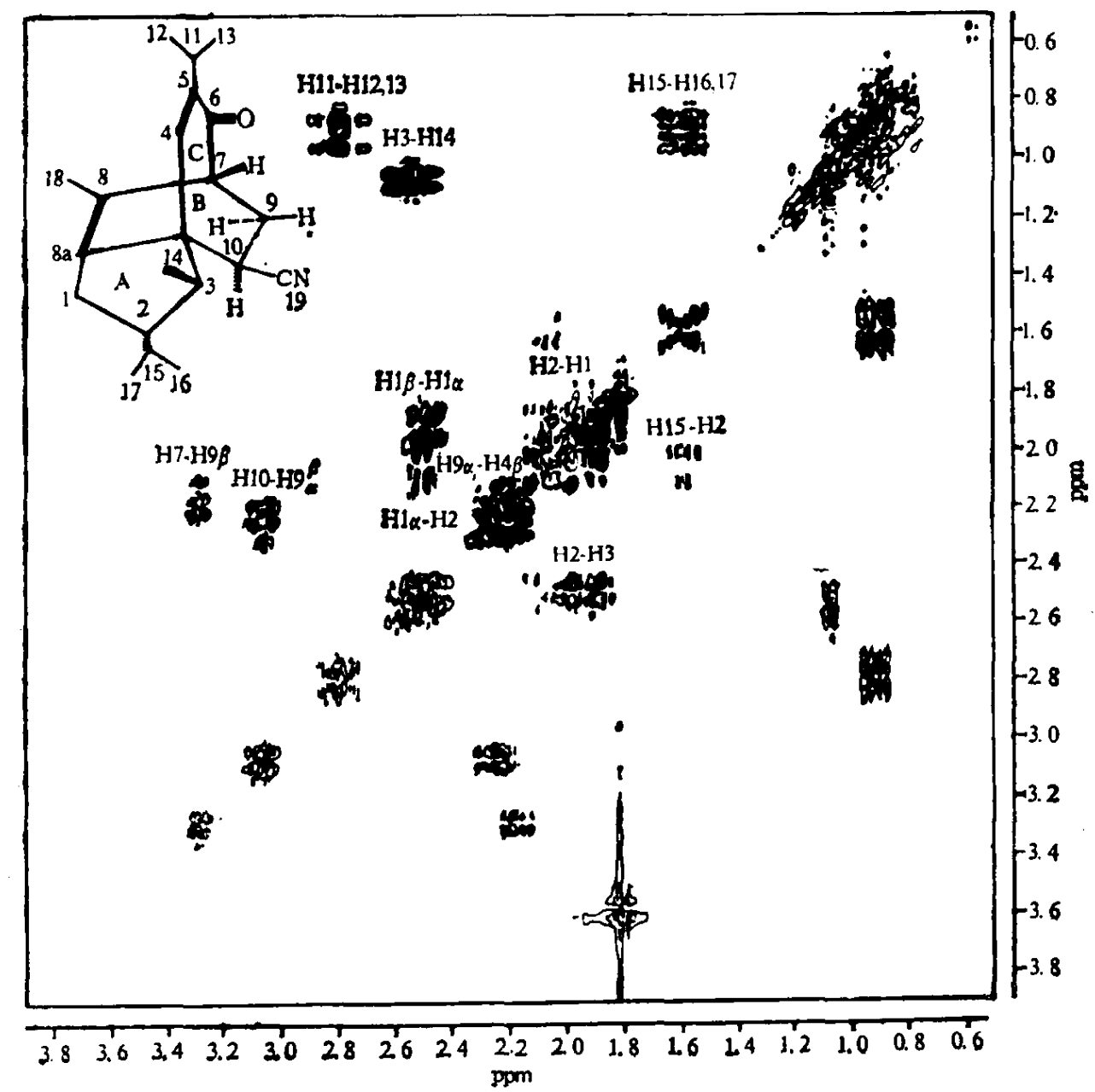

图 1 化合物相敏 COSY 谱

推出一些子结构,异核远程相关谱的交叉蜂提供相距一键和两键的官能团信息,成为结构分析, 特别是确定季碳的相邻官能团的有力证据. 如 $\delta_{\mathrm{C}} 196.06 \mathrm{ppm}$ 酮碳的远程相关峰 $0_{\mathrm{H}} 6.59 \mathrm{ppm}$ $\left({ }^{3} J_{\mathrm{CH}}\right), \delta_{\mathrm{H}} 3.26 \mathrm{ppm}\left({ }^{2} J_{\mathrm{CH}}\right)$ 和 $\delta_{\mathrm{H}} 2.15 \mathrm{ppm}\left({ }^{3} J_{\mathrm{CH}}\right) ; \delta_{\mathrm{c}} 121.57 \mathrm{ppm}$ 氛基碳的远程相关峰 $\delta_{\mathrm{H}} 2.15$ 和 $2.22 \mathrm{ppm}$ 以及 $\delta_{\mathrm{H}} 3.04 \mathrm{ppm}$; 高场季碳 $\delta_{\mathrm{c}} 53.85 \mathrm{ppm}$ 与 $\delta_{\mathrm{H}} 3.04$ 和 $6.59 \mathrm{ppm}$ 的交叉蜂等, 可以推出 子.结构 C 环。综合分析所有 ${ }^{33} \mathrm{C}$ 和 ${ }^{1} \mathrm{H}$ 化学位移, 偶合分裂以及季碳 $\delta_{\mathrm{c}} 145.56$ 和 $\delta_{\mathrm{c}} 144.74$ 


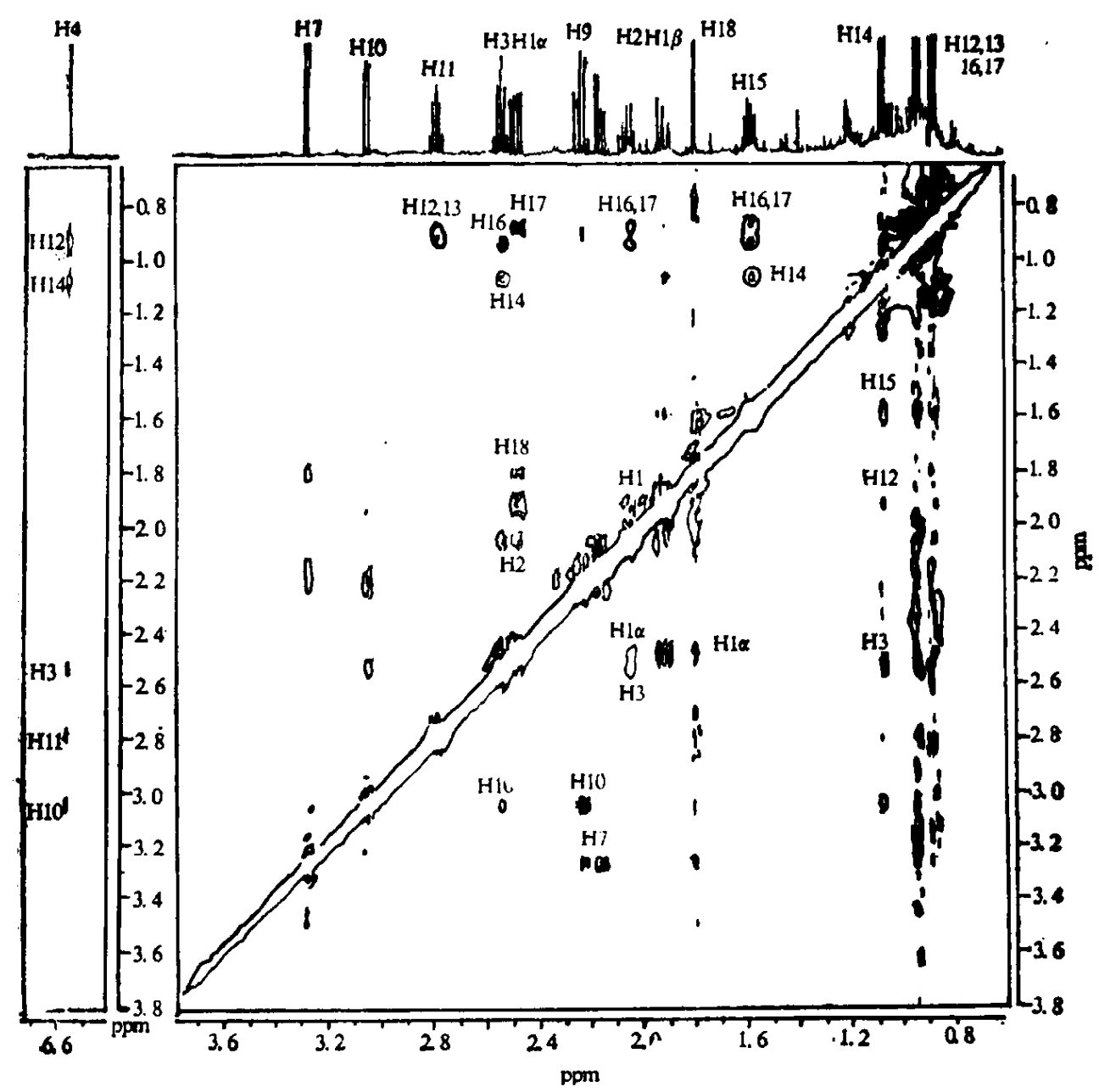

图 2 相敏 NOESY 谱

$\mathrm{ppm}$ 的远程 $\mathrm{C}-\mathrm{H}$ 相关峰, 化合物的平面结构得以确定, 它是一个与五元环椆合的六元、七元 环桥连的三环化合物, 依靠 NOE 峰和远程 $\mathrm{C}-\mathrm{H}$ 相关峰的分析确定其立体结构. H 3 和 $\mathrm{H} 10, \mathrm{HK}$ 和 $\mathrm{CH} 3-14$ 的强 $\mathrm{NOE}$ 峰(图 2)表明, $\mathrm{CH}^{3}-14$ 与 $\mathrm{C} 3 \mathrm{a}-\mathrm{C} 4$ 键顺式、H3 与 $\mathrm{H} 10$ 空间接近; $\mathrm{C} 8 \mathrm{a}-\mathrm{H} 7$ 和 $\mathrm{C} 10-\mathrm{H} 7$ 的强三键交叉峰表明 $\mathrm{C} 3 \mathrm{a}$ 和 $\mathrm{C} 10$ 分别与 $\mathrm{H} 7$ 处于反向构 象. $\mathrm{H} 9$ 质子的两组峰, 除了 ${ }^{2} J_{\mathrm{gem}}=14.55 \mathrm{~Hz}$ 外, $\delta_{\mathrm{H}} 2.22 \mathrm{ppm}$ 峰含有 $J_{1}=9.70 \mathrm{~Hz}$ 和 $J_{2}=$ $1.44 \mathrm{~Hz}$ 的分裂, 而 $\delta_{\mathrm{H}} 2.15 \mathrm{ppm}$ 却有 $\mathrm{J}_{\mathrm{1}}-6.40$ 和 $\mathrm{J}_{2}=2.81 \mathrm{~Hz}$ 的分裂. 以上数据均符合以 五元环为平面, $\mathrm{CH}$ 3-14 为 $\beta$ 取向, 桥碳 3a 的直立键构成七元环, 六元环为船式构象, $\mathrm{C} 10$ 上 的彺基为 $\beta$ 取向, $\delta_{\mathrm{H}} 2.22 \mathrm{ppm}$ 为 $\mathrm{H} 9(\alpha)$ 共振, 其船式构象的桥碳上 $\mathrm{H} 7$ 与 $\mathrm{H} 9(\alpha)$ 具有 较小偶合常数 $\left({ }^{3} \mathrm{~J}_{\mathrm{H} 7-\mathrm{H} 9 \mathrm{a}}-1.44 \mathrm{~Hz}\right)$; 在此基础上确定 $\mathrm{C} 2$ 上异丙基的取向, 由模拟谱得到 $3^{3} J_{\mathrm{H}_{2}-\mathrm{H} 3}-7.0 \mathrm{~Hz}$ 而五元环的顺式相邻质子偶合常数偏大(约 $10 \mathrm{~Hz}$ ), 则 $\mathrm{H} 3$ 和 $\mathrm{H} 2$ 必处于 反向, $\mathrm{CH}$ 3-14 取 $\beta$ 构象, C-2 上异丙基则取 $\alpha$ 构象; 由相邻质子 $J$ 值分析, 归属了磁非等 价的两个 $\mathrm{Hl}$ 质子, $\mathrm{Hl}(\alpha)$ 与 $\mathrm{CH}_{3}-17 、 \mathrm{CH}^{3}-18$ 有 $\mathrm{NOE}$ 峰, 表明 $\mathrm{Hl}(\alpha)$ 与异丙基同 向; $\mathrm{H} 1(\beta)$ 与 $\mathrm{CH} 3-14$ 有 $\mathrm{NOE}$ 峰等均支持上述构象分析, 且也符合生成此化合物的原料 找术醇的构象. $\mathrm{HI}(\alpha)$ 与 $\mathrm{CH}$ 3-17 有 $\mathrm{NOE}$ 而与 $\mathrm{CH}^{3-16}$ 无 NOE, 反之, $\mathrm{H} 3$ 与 $\mathrm{CH}^{3}-$ 16 有 NOE, 与 $\mathrm{CH}^{3}-17$ 无 NOE, 这表明 $\mathrm{C} 2$ 上异丙基的优势构象是 $\mathrm{H} 2$ 与 $\mathrm{H} 15$ 顺式, $\mathrm{CH} 3-17$ 与 $\mathrm{Hl}(\alpha), \mathrm{CH}^{3}-16$ 与 $\mathrm{H} 3$ 在空间接近, 其结构为 $3(\mathrm{R}), 8$-二甲基-2(R), $5-$ 二 
（1-甲基-乙基)-6-间-2,3,6,7,9,10-六氢-1H-3A,7-乙真-10-腈（结构式见图 1).

'HNMR 谱中 $\mathrm{CH}^{3}-18(1.79 \mathrm{ppm})$ 有 $1.44 \mathrm{~Hz}$ 裂分, 由模拟谱可知它来源于 ' $J_{\left(\mathrm{CH}_{3}\right)}$ 28- $\mathrm{H} 18-$ $2.0 \mathrm{~Hz}$ 和 ${ }^{3} \mathrm{~J}_{\left(\mathrm{CH}_{3}\right) \text { ls }-\mathrm{His}}-0.4 \mathrm{~Hz}$, 为顺式开烯丙基远程偶合, 同样也发现了 $\mathrm{H} 4$ 与 $\mathrm{H} 11$ 的烯 丙基远程偶合 $(2 \mathrm{~Hz})$.

由结构分析和谱线归属发现,通过二键和三键的异核 $\mathrm{C}-\mathrm{H}$ 相关峰不仅用做结构确定, 环 与取代基构象分析,对谱线归属，特别是那些用屏蔽效应难以解释的季碳归属可提供十分重要 的证据. 除酮碳外,此化合物在低场有四个季碳峰 $(\mathrm{C} 8, \mathrm{C} 8 \mathrm{a}, \mathrm{C5}, \mathrm{C19})$. 与 $\mathrm{CH} 3-12,13$ 有远程 交叉峰的 $\delta_{\mathrm{c}} 144.65 \mathrm{ppm}$ 必定归属为与异丙基相连的 C5 谱线; 与 $\mathrm{H} 9$ 和 $\mathrm{H} 10$ 有远程交叉峰 的 $\delta_{\mathrm{c}} 121.57 \mathrm{ppm}$ 为保基碳峰; 余下的 C8 和 C8a 碳峰,具有一些相同的交叉峰, 而 ${ }^{3} J_{\mathrm{Cs}-\mathrm{H} 10}$ 和 ${ }^{3} J_{\mathrm{C} 8-\mathrm{H} 9}$ 交叉峰为有力证据, 确定 $\mathrm{C} 8 \mathrm{a}$ 在低场, $\mathrm{C} 8$ 共振在高场.

${ }^{13} \mathrm{CNMR}$ 数据: $\mathrm{C} 1$ : 31.08ppm;C2 :48.16ppm;C3:43:05ppm;C3a :53.85ppm;C4:1 40.75ppm: C5: $144.65 \mathrm{ppm} ; C 6: 196.06 \mathrm{ppm} ; \mathrm{C7}: 56.28 \mathrm{ppm} ; C 8: 123.40 \mathrm{ppm} ; C 8 \mathrm{a}: 145.56 \mathrm{ppm} ; C 9: 27.78 \mathrm{ppm}$; $\mathrm{C10}$ :34.66ppm;C11:28.17ppm;C12:22.08ppm;C13:21.48ppm;C14:11.74ppm;C15:28.27ppm; C16:21.64ppm; C17:21.31ppm;C18:18.30ppm;C19:121.57ppm.

${ }^{1} \mathrm{H}$ NMR 谱数据: $\mathrm{H} 1 \beta$ : 1.90 和 $\mathrm{H} 1 \alpha: 2.47 \mathrm{ppm}\left({ }^{2} J_{\mathrm{gem}}-14.77 \mathrm{~Hz},{ }^{3} J_{\mathrm{H}_{1} \beta-\mathrm{H}_{2}}-11.50 \mathrm{~Hz}\right.$, $\left.{ }^{3} J_{\mathrm{H} 1 a-\mathrm{H}_{2}}=6.30 \mathrm{~Hz},{ }^{3} J_{\mathrm{H} 1 \beta-\mathrm{H} 18}=2.00 \mathrm{~Hz},{ }^{5} J_{\mathrm{Hla}-\mathrm{H} 18}=0.40 \mathrm{~Hz}\right) ; \mathrm{H} 2: 2.05 \mathrm{ppm}\left({ }^{3} J_{\mathrm{H}_{2}-\mathrm{H} 3}-7.00 \mathrm{~Hz}\right.$, $\left.{ }^{3} J_{\mathrm{H}_{2}-\mathrm{H} 15}-9.70 \mathrm{~Hz}\right) ; \mathrm{H} 3: 2.53 \mathrm{ppm}\left({ }^{3} J_{\mathrm{HS}-\mathrm{H} 14}-7.25 \mathrm{~Hz}\right) ; \mathrm{H} 4: 6.59 \mathrm{ppm}\left({ }^{4} J_{\mathrm{H} 4-\mathrm{H} 11}-2.00 \mathrm{~Hz}\right) ; \mathrm{H} 7:$ $3.26 \mathrm{ppm}\left({ }^{3} J_{\mathrm{H7}-\mathrm{H} 9 \beta}-6.40 \mathrm{~Hz},{ }^{3} J_{\mathrm{H} 7-\mathrm{H} 9 \alpha}-1.44 \mathrm{~Hz}\right) ; \mathrm{H} 9 \alpha: 2.22 \mathrm{ppm}$ 和 $\mathrm{H} 9 \beta: 2.15 \mathrm{ppm}\left({ }^{2} J_{\mathrm{sem}}=\right.$ $14.55 \mathrm{~Hz},{ }^{3} J_{\mathrm{H} 9 \alpha-\mathrm{H} 10}-9.70 \mathrm{~Hz},{ }^{3} J_{\mathrm{H} 9 \mathrm{~B}-\mathrm{H} 10}-2.81 \mathrm{~Hz}$ ); $\mathrm{H} 10: 3.04 \mathrm{ppm} ; \mathrm{H} 11: 2.77 \mathrm{ppm}$ (七重峰, $\left.{ }^{3} \mathrm{~J}-6.86 \mathrm{~Hz}\right) ; \mathrm{H} 12 、 13: 0.877$ 和 $0.920 \mathrm{ppm}\left(d,{ }^{3} \mathrm{~J}-6.86 \mathrm{~Hz}\right) ; \mathrm{H1} 4: 1.11 \mathrm{ppm}\left(d,{ }^{3} \mathrm{~J}-7.25 \mathrm{~Hz}\right)$; H15: $1.57 \mathrm{ppm}\left(m,{ }^{3} \mathrm{~J}-6.51 \mathrm{~Hz}\right) ; \mathrm{H} 16 、 17: 0.93$ 和 $0.865 \mathrm{ppm}\left(d,{ }^{3} \mathrm{~J}-6.51 \mathrm{~Hz}\right) ; \mathrm{H} 18$ : $1.79 \mathrm{ppm}$ $\left(d d, J_{1}-2.0 \mathrm{~Hz}, J_{2}=0.4 \mathrm{~Hz}\right)$.

[1] Guo Yongtien et al., Acta Pharmacentica Sinica (in press).

[2] Marion \& Wüthrich, BBRC, 1983, 113, 967.

[3] Bodenhausen, G., Kogler, K. and Ernst, R. R., J. M. R., 53 (1984). 370.

[4] Wilde, J. A. et al., J. M. R., 59 (1984), 343.

[5] Martin, G. E. and Zektzer, A. S., Texas A8cM Newslezer, 1987, 344-52.

[6] Zektzer, A. S. ot sl., Mag*. Reson. Chem., 25 (1987), 752-756.

[7] Salazar, M., Zextcer, A. S. and Martin, G. E., ibid, 26 (1988), 24-27. 